Sessions d'étude - Société canadienne d'histoire de l'Église catholique

\title{
La première Caisse ecclésiastique du clergé canadien
}

\section{Lucien Lemieux}

Volume 44, 1977

URI : https://id.erudit.org/iderudit/1007126ar

DOI : https://doi.org/10.7202/1007126ar

Aller au sommaire du numéro

Éditeur(s)

Les Éditions Historia Ecclesiæ Catholicæ Canadensis Inc.

ISSN

0318-6172 (imprimé)

1927-7067 (numérique)

Découvrir la revue

Citer cet article

Lemieux, L. (1977). La première Caisse ecclésiastique du clergé canadien.

Sessions d'étude - Société canadienne d'histoire de l'Église catholique, 44, 5-22.

https://doi.org/10.7202/1007126ar

Tous droits réservés @ Les Éditions Historia Ecclesiæ Catholicæ Canadensis Inc., 1977
Ce document est protégé par la loi sur le droit d'auteur. L'utilisation des services d'Érudit (y compris la reproduction) est assujettie à sa politique d'utilisation que vous pouvez consulter en ligne.

https://apropos.erudit.org/fr/usagers/politique-dutilisation/ 


\section{La première Caisse ecclésiastique du clergé canadien}

\section{INTRODUCTION}

Les revenus du clergé fluctuent selon les époques et sont toujours tributaires de la situation sociale. Le passage du régime français au régime britannique, la diminution du nombre des prêtres, les hauts et les bas des récoltes dont dépendaient les dîmes, les montants d'argent versés en honoraires de messes, les tarifs de certains actes sacramentels ou pastoraux tels que les mariages ou les funérailles, autant de facteurs qui ont eu de l'influence sur les moyens de subsistance des prêtres séculiers à la fin du $19^{\mathrm{e}}$ siècle.

Si dans l'ensemble les prêtres pouvaient ainsi réussir à vivre, en général au même diapason que leurs paroissiens, ils se trouvaient cependant à l'occasion dans le besoin, en particulier lors d'une maladie ou encore au moment de la retraite.

Il est par ailleurs important de constater jusqu'à quel point des membres du clergé étaient sensibles à la possibilité de partager une partie de leur avoir en vue de s'entraider et même de rendre service ensemble à telle personne ou à tel groupe de personnes. L'histoire de la première Caisse ecclésiastique se comprend dans ce sens et dans cet esprit.

\section{I - FONDATION ET PARTICIPATION}

\section{Consultations}

En 1793, le curé de Longueuil, $\mathbf{M}^{* r}$ Pierre Denaut, récemment choisi comme évêque coadjuteur du diocèse de Québec, s'entretint avec ses confrères de la rive sud de Montréal sur un projet de Caisse ecclésiastique. L'idée fut accueillie avec chaleur, et les curés présents montrèrent les dispositions les plus favorables. $\mathbf{M}^{\mathrm{gr}}$ Denaut se chargea de mener ce projet à terme. Il ne doutait pas de l'empressement que les prêtres mettraient à participer à une telle entraide mutuelle; le clergé en tirerait aussi un grand honneur, 
selon lui ${ }^{1}$. Il attendit cependant le meilleur moment pour parler de son projet à $\mathbf{M}^{\text {gr }}$ Jean-François Hubert, évêque de Québec. Celui-ci fut mis au courant en décembre 1795. Il s'intéressa tout de suite au projet, mais avant de publier la circulaire qu'il avait commencé à écrire sur le sujet, il désirait connaître l'opinion des membres du clergé de tout le diocèse, par l'intermédiaire des archiprêtres. $\mathrm{M}^{\mathrm{gr}}$ Hubert était prêt à constituer une Caisse ecclésiastique pour les besoins les plus pressants, mais il croyait opportun d'élargir la consultation avant de publier une constitution élaborée ${ }^{2}$.

L'évêque adressa une lettre à ses archiprêtres dès le 12 janvier 1796. Il requérait leur collaboration pour amener leurs confrères à former un "fonds de charité, monument de bienfaisance et source de mille biens ». $\mathbf{M}^{\mathrm{gr}}$ Hubert était par ailleurs peiné que des prêtres, appelés sans aucun doute à servir de modèles, aient laissé à leur sort des sommes d'argent qu'ils auraient beaucoup mieux fait de distribuer de leur vivant. Il attendait donc une réponse à chacune des trois questions suivantes :

1. Une Caisse du Clergé est-elle à propos?

2. Quels pourraient en être l'objet et l'étendue ?

3. Quelle forme d'administration conviendrait-il ?

Sept archiprêtres sur vingt-trois n'avaient pas encore répondu à leur évêque quatre mois plus tard. $\mathbf{M}^{\mathrm{wr}}$ Hubert n'envoya pas moins à chacun une nouvelle lettre dans laquelle il développa son plan d'établissement d'une telle Caisse. Il incita son coadjuteur à y souscrire le premier ainsi qu'à stimuler les archiprêtres de la partie sud du diocèse à bien exécuter leur travail. Il le prévint que des prêtres auraient voulu que la contribution de chacun équivalût à $1 / 26^{\circ}$ de ses revenus, mais $M^{\mathrm{gr}}$ Hubert s'en tint à $1 / 50^{\circ}$, quitte à ce que l'autre proposition fût réalisée plus tard ${ }^{4}$.

L'évêque de Québec présenta un plan qui résultait des réponses des prêtres ainsi que de son expérience et de ses réflexions person-

1 AAQ (Archives de l'archidiocèse de Québec), CL (Copie de lettres), V, p. 441, 10 décembre 1795, Denaut à Hubert. à Denaut.

2 AAQ, RL (Registres des lettres), II, p. 242, 14 décembre 1795,

3 MEQ (Mandements, lettres pastorales et circulaires des évêques de Québec), II, Québec, A. Côté et Cie, 1888, p. 489.

4 AAQ, RL, II, pp. 270-271, 5 mai 1796. 
nelles. Il énuméra onze objectifs à atteindre grâce à cette Caisse, tout en reconnaissant qu'il faudrait les répartir et les réaliser par étapes :

- fournir un supplément de revenus à l'évêque coadjuteur ;

- défrayer l'envoi et la subsistance des prêtres dans les missions et les paroisses qui en étaient dépourvues ;

- permettre à des prêtres de continuer à rendre service là où les gens ne pouvaient les soutenir financièrement ;

- assurer une retraite honorable aux prêtres infirmes ;

- payer une pension de collégiens et d'ecclésiastiques dont les parents étaient pauvres ;

- doter des filles pauvres désirant devenir religieuses;

- secourir les communautés religieuses indigentes ;

- soulager les incendiés, les naufragés et autres victimes de ce genre ;

- favoriser l'ensemencement des terres lors des années de disette ;

- participer à l'établissement d'écoles et à l'enseignement du catéchisme dans les paroisses fort étendues ou fort peuplées ;

- collaborer à toute bonne œuvre.

L'ensemble des prêtres serait divisé en douze cantons (cinq dans chacun des districts de Québec et de Montréal, et deux dans celui de Trois-Rivières) et un curé serait élu dans chacun d'entre eux comme syndic. L'évêque se réservait le droit de choisir l'élu dans les cas où deux prêtres (ou plus) auraient un nombre égal de votes. Deux mois plus tard, les syndics de chaque canton seraient appelés à élire un représentant de district. Une fois l'an, le $1^{\mathrm{er}}$ juillet, les membres paieraient en argent, au prix courant de la saison, $1 / 50^{\mathrm{e}}$ des minots de tous grains perçus en dîme depuis une année. Le syndic, auquel chaque curé aurait affaire dans son canton, accepterait par la même occasion des legs, des donations, des présents ou tout autre cadeau pour la Caisse ecclésiastique. Le $1^{\text {er }}$ août, le syndic général (le grand responsable au niveau d'un district) devrait avoir reçu le compte exact de tous les versements. Il lui appartiendrait de régler alors les cas difficiles ou de les référer au Bureau général de la Caisse qui se réunirait annuellement en septembre.

Le Bureau inclurait l'évêque et son coadjuteur, les trois syndics généraux, les vicaires généraux et possiblement trois autres prêtres choisis par l'évêque. On rendrait alors compte des recettes, déterminerait l'emploi des deniers et délibérerait sur toutes les autres 
affaires de la Caisse. Les syndics généraux informeraient ensuite leurs syndics particuliers, qui, eux, se chargeraient d'en entretenir leurs curés respectifs ; ils pourraient en même temps les consulter sur des changements à effectuer l'année suivante. Le Bureau jouirait du droit de tenir des réunions spéciales ; en cas d'impossibilité de réunion, l'évêque pourrait octroyer de l'argent par lui-même ou mieux en accord avec le syndic général de la région concernée, pour autant qu'il le prît à même le montant qui lui serait alloué annuellement pour des cas spéciaux. Le remplacement des syndics s'opérerait par élection après la mort ou la démission de l'un ou de l'autre. "Le temps et l'expérience, en mûrissant nos idées sur cette Caisse, nous fourniront, je l'espère, des moyens de l'améliorer et d'en étendre les objectifs et les règles d'administration ${ }^{5}$.

Les commentaires ne tardèrent pas à surgir. L'évêque coadjuteur se fit lui-même l'interprète de plusieurs curés de son district : «....tous veulent une Caisse ecclésiastique, mais avec restriction à l'étendue de l'objet et amendement sur la formation et l'administration de la Caisse ». Il précisa qu'il s'agissait d'abord de s'en tenir à deux objectifs : une retraite honorable aux prêtres et un supplément au coadjuteur. Compte tenu du capital amassé, on devrait ensuite aider à la subsistance des prêtres dans le besoin, puis payer la pension à des ecclésiastiques pauvres; on pourrait même, en dernier lieu, déffrayer le transport de prêtres étrangers, désireux d'exercer leur ministère au pays. En somme, il ne s'agissait pas d'une Caisse publique, mais ecclésiastique ; les objectifs qui ne concernaient pas les prêtres devaient être mis de côté. De toute façon, il fallait premièrement s'occuper de remplir les coffres de la Caisse; les moyens de la vider ne manqueraient jamais. Quant au choix du syndic général, on préférait qu'il dépendît de l'élection de tous les membres d'un même district, et non seulement de celle des syndics particuliers ; autrement, dans le district de Trois-Rivières où se trouvaient seulement deux syndics particuliers, il serait de fait revenu à l'évêque de choisir le syndic général. De plus, pourquoi ne pas élire des syndics particuliers pour un mandat de deux ou de trois ans, au lieu de les élire de façon indéfinie ? À propos du montant d'argent à verser en cotisation, il faudrait le fixer pour chaque espèce de grains (par cent minots, par exemple), car les prix changeraient selon les régions. Finalement, chaque syndic général ne gagnerait-il pas à rendre ses comptes dans son propre district? La réunion serait alors présidée par l'évêque à Québec,

$5 \quad M E Q$, II, pp. 490-495, 4 mai 1796. 
par son coadjuteur à Montréal, par son vicaire général à TroisRivières. En feraient partie, non seulement les syndics particuliers, mais aussi tous les contribuables qui le désireraient; ils seraient nombreux si cette rencontre coïncidait avec la fête du sacerdoce, le premier jeudi après le 29 août. La reddition des comptes et les délibérations seraient alors communiquées, et tout serait décidé à la majorité des voix, sans que de longs voyages à Québec ou ailleurs ne fussent nécessaires ${ }^{6}$.

Le projet de $\mathrm{M}^{\mathrm{gr}}$ Hubert était ainsi fort modifié. Bien que des prêtres aient continué à lui faire part de leur adhésion au plan initial (certains revenaient alors des missions où ils avaient été sans nouvelles depuis près d'un an) ${ }^{7}$, il resta ouvert au nouveau plan que lui fit parvenir son coadjuteur à la suite des commentaires de nombreux curés ${ }^{8}$. Ce n'était pas de son goût, mais il consentit à l'adopter uniquement parce qu'il venait de $\mathrm{M}^{\mathrm{gr}^{\mathrm{r}}}$ Denaut ${ }^{9}$. De fait, $\mathbf{M}^{\mathrm{gr}}$ Hubert n'y donna pratiquement pas suite. Il démissionnerait d'ailleurs quelques mois plus tard et mourrait peu après. De son côté, $\mathrm{M}^{\mathrm{gr}}$ Denaut fut tellement occupé par sa cure qu'il conserva et par sa nouvelle tâche épiscopale, qu'il délaissa momentanément l'organisation de la Caisse ecclésiastique.

\section{Accroissement progressif des membres}

Bien que fondée en 1796, la Caisse ou mieux, selon son nom officiel, la Société ecclésiastique Saint-Michel prit vraiment son envol en 1799, au presbytère de la paroisse Saint-Michel, près de Québec. L'hôte, François-Joseph Deguise, était d'ailleurs l'un des douze premiers à y avoir signé son adhésion. Le curé de Québec, JosephOctave Plessis, alors désigné comme évêque coadjuteur, était aussi de ce nombre. Il semble même avoir été l'instigateur de la rencontre; il en sortit d'ailleurs président de la Société ${ }^{10}$.

On note cependant quelques manifestations de méfiance. Des prêtres de la région de Montréal songent même à avoir leur propre caisse. Le curé Joseph Lelièvre-Duval de Repentigny fut en effet

6 AAQ, $C L$, V, pp. 459-461, 23 mai 1796.

7 AAR (Archives de l'archevêché de Rimouski), Rimouski (Cathédrale), 1763-1800, 5 août 1796, Pierre Robitaille, missionnaire à Portneuf, à Hubert.

8 AAQ, $C L$, V, pp. $479-480,12$ octobre 1796.

9 AAQ, RL, II, pp. 313-314, 27 octobre 1796, à Denaut.

10 Écho du pays, 2 juillet 1835 ; J.B.A. FerLAND, Mgr Joseph-Octave Plessis, évêque de Québec, Québec, 1878, p. 44 ; Victor MoRIN, Syndicalisme et Mutualité, dans Les Cahiers des Dix, 24 (1959), p. 76. 
vexé du fait qu'on avait laissé de côté neuf lettres reçues trop tard, lors de l'assemblée de 1803. Selon lui, le délai fixé n'avait pas été assez long. L'élection des syndics devait donc, à son avis, être reprise. "Je serai fidèle et constant; mais je ne serai jamais d'avis qu'on prive aucun membre des droits qu'il a dans la Société, avant que les autres l'aient unanimement condamné à les perdre »"11. Mais le tout se régla à l'amiable et le projet d'une seconde caisse s'estompa. Un autre projet semblable apparaîtrait plus tard à Sainte-Anne-dela-Pocatière. Le curé Charles-François Painchaud, déçu de la présidence à perpétuité exercée par $\mathbf{M}^{\mathrm{gr}}$ Plessis de même que du peu de considération apportée aux prêtres eux-mêmes par les responsables de la Caisse, essaya de fonder la Société sacerdotale du Clergé. Mais trop peu de prêtres y adhérèrent, ce qui l'empêcha d'opérer ${ }^{12}$.

Entre-temps, d'autres membres s'adjoignirent à la Caisse ecclésiastique, tel Laurent Aubry en 1806, qui promit de remplir exactement toutes les obligations que la dite association lui prescrirait ${ }^{13}$, tel encore Jean-Baptiste Allain, missionnaire aux Îles de la Madeleine ; celui-ci serait désormais tenu, le $1^{\text {er }}$ juillet de chaque année, de verser à la Caisse $1 / 50^{\mathrm{e}}$ de ses revenus ecclésiastiques, à l'exception cependant de ses honoraires de messes et de tout casuel provenant de sa mission ${ }^{14}$. En 1816, Joseph-Marie Morin était prêt à signer une formule d'inscription, mais il avait une copie qui datait de 1802 ; avait-on exigé une autre façon de procéder depuis lors ? ${ }^{15}$ Le nombre des membres s'élevait alors à $94{ }^{16}$.

L'intérêt individuel y était parfois apparent, ce qui donnait alors l'impression d'une assurance-maladie. Exerçant son ministère à Yamaska, Jean Holmes motiva ainsi sa demande d'entrée à la Caisse : "... c'est que si je viens à me casser dans les chemins dangereux de ma mission, on payera pour moi, et si je ne me

11 ACAM (Archives de la chancellerie de l'archevêché de Montréal), 355.105, 12 septembre 1803, à Plessis.

12 ASN (Archives du Séminaire de Nicolet), Succession Bois, D, Manuscrits : Documents historiques, IX, p. 45, 2 janvier 1820.

13 ADSJQ (Archives du diocèse de Saint-Jean-de-Québec), Contrecoeur, 4, A-16, 15 décembre 1806, à Plessis.

14 AAQ, $R L$, VI, p. 268, 22 octobre 1808, Plessis à Allain.

15 AEP (Archives de l'évêché de Sainte-Anne-de-la-Pocatière), Montmagny (St-Thomas), I, p. 92, 26 mars.

16 AAQ, $R L, \mathrm{IX}$, p. 37, octobre 1816, Plessis à Joseph-Marie Bellenger, missionnaire à Carleton, Baie des Chaleurs. 
casse que les jambes, on ne me fera pas crever de faim 》17. $M^{g r}$ Plessis conseillait d'ailleurs lui-même à certains curés de ne pas négliger leur contribution à la Caisse ; celle-ci serait leur seule ressource en cas d'infirmité. Il précisa ainsi à Joseph Marcoux, missionnaire à Saint-Régis, la manière de calculer sa cotisation ${ }^{18}$. "Vous aurez tort de quitter la Caisse », écrivait-il à Jean-Baptiste Marchand, curé de Sandwich ; "votre vieil âge approche et ce sera votre seule ressource temporelle ${ }^{19}$. Le $1 / 50^{\mathrm{e}}$ des revenus correspondait parfois à un montant dérisoire, par exemple dans le cas de Sévère-Joseph-Nicolas Dumoulin, missionnaire dans le NordOuest. Sa contribution était de $\$ 2.00$ pour ses revenus de Pembina et d'un écu pour la Fourche ${ }^{20}$.

Pour sa part, $\mathbf{M}^{\text {gr }}$ Jean-Jacques Lartigue, évêque auxiliaire de Montréal, incita ses jeunes prêtres à s'associer à la Caisse ecclésiastique ; ce serait pour eux une ressource indispensable et assurée, si l'âge et les infirmités les rendaient incapables d'exercer le saint ministère ${ }^{21}$. Ignace Bourget, surpris que Narcisse-Charles Fortier fût membre de la Société, lui avoua avoir cru que les secrétaires d'évêque en étaient exclus; il chercha à s'y joindre lui aussi 22 . De fait, entre 1825 et 1829 , on compte en moyenne huit nouvelles entrées par année ${ }^{23}$. Le refus d'admission pour des raisons «pas trop connues » aux dires de Pascal Pouliot, vicaire à Rimouski, ne le découragea pas ; ce devait être une question de règlement mal appliqué ; il réitéra sa demande l'année suivante 24 et il fut accepté.

Des cas particuliers rendaient parfois la situation un peu enchevêtrée. Ainsi, lors de la mésentente entre $\mathrm{M}^{\mathrm{gr}}$ Lartigue et le curé Augustin Chaboillez de Longueuil ${ }^{25}$, il se tint une élection des

17 AEN (Archives de l'évêché de Nicolet), St-Frédéric, I, 8 juillet 1825, à Plessis.

18 AAQ, $R L$, IX, p. 191, août 1817.

19 Ibid., p. 516, 27 mars 1819.

20 AAQ, Rivière Rouge, II, p. 176, 13 décembre 1822, à Plessis.

21 ACAM, RLL (Registres des lettres de $M^{g r}$ Lartigue), IV, p. 340, 28 avril 1828, à Gabriel-Léandre Arsenault, vicaire à la Pointe-aux-Trembles.

22 ASQ (Archives du Séminaire de Québec), Lettres T, p. 113, 24 décembre 1824 .

23 ACAM, 295.099; 830-6, 1er mai, Panet aux membres de la Caisse.

24 AAR, Rimouski (Cathédrale) 1801-1848, 12 septembre 1832. à Charles-Félix Cazeau.

25 L. LEMIEUX, L'établissement de la première province ecclésiastique au Canada, 1783-1844, Montréal, Fides, 1968, pp. 164-166 et pp. 174-183. 
procureurs de la Caisse ecclésiastique. $\mathbf{M}^{\mathrm{gr}}$ Lartigue entendit dire alors que son opposant n'avait pas été réélu et qu'il s'était même retiré de la Société pour encourager l'Éducation Ecclésiastique 26 pour des motifs que même ses amis avaient réprouvés ${ }^{27}$. Les résultats d'élection n'étaient cependant pas encore connus. $\mathrm{M}^{\mathrm{gr}}$ Plessis ne souhaitait pas moins que Chaboillez fût exclu de la Caisse ${ }^{28}$. De fait, le curé de Longueuil n'arriva pas parmi les douze premiers, quoiqu'il ait eu plus de suffrages que ne l'avait prévu l'évêque de Québec ${ }^{29}$. Un curé en instance de se retirer du ministère pouvait-il devenir membre de la Caisse, quitte à ce qu'il payât $1 / 100^{\mathrm{e}}$ de ses revenus depuis qu'il avait été ordonné prêtre ${ }^{30}$ ? $\mathbf{M}^{\text {gr }}$ Signay promit d'examiner le cas, tout en se demandant si ce bon curé François Boissonnault, une fois retiré, ne pourrait pas rendre service au Séminaire de Nicolet par ses conférences ou autrement ${ }^{31}$; il aurait alors moins besoin des prestations de la Caisse.

Il ressort clairement qu'aucun prêtre n'était obligé de faire partie de la Caisse Saint-Michel, mais que la plupart des jeunes y adhéraient tout naturellement. Les évêques semblaient les y inviter assez instamment. Par ailleurs, personne n'en devenait membre, sans y être admis officiellement par le Bureau de direction. C'était sûrement plus simple dans le cas des nouveaux prêtres, car ils assuraient la Caisse de plusieurs années de cotisation.

\section{II - SERVICES RENDUS}

\section{Priorité aux prêtres malades ou âgés}

Malgré un début lent, où l'on se préoccupa d'accroître le nombre des membres et de recueillir les cotisations, la Caisse SaintMichel fut vite appelée à rendre service. Le curé Antoine Valade de Sainte-Marie de Beauce postula une offrande dès l'été $1799{ }^{32}$; mais ce fut en vain. À l'automne $1800, M^{\text {gr }}$ Plessis demanda à $\mathrm{M}^{\mathrm{gr}}$ Denaut si «les associés de notre petite Caisse ecclésiastique,

26 Il s'agit d'une Société fondée à Varennes le 4 juillet 1822 et constituée de prêtres religieux désireux d'aider financièrement des jeunes à poursuivre leurs études en vue de réaliser leur vocation sacerdotale (ASQ, Polygraphie 19 , p. 30,4 juillet 1822 ).

27 ACAM, RLL, III, 55, 2 août 1824, à Plessis.

28 ACAM, 295.101, 824-32, 16 août, à Lartigue.

29 ACAM, 295.101, 824-38.

30 ACAM, RLL, VII, 344, 19 décembre 1833, à Signay.

31 ACAM, 295.101, 833-109, 24 décembre 1833, à Lartigue.

$32 \mathrm{AAQ}, 61$ CD, Sainte-Marie de Beauce, I, p. 66, 14 juillet, à Plessis. 
qui n'a encore rien déboursé, seraient flattés d'entreprendre » comme première bonne cuvre le paiement de l'hospitalisation de James MacDonell. Ce missionnaire de la Nouvelle-Écosse, martyr de son zèle, était tombé depuis une couple d'années dans un état de démence et d'infirmité. Il faudrait le faire venir à Québec, car personne ne pouvait s'en occuper en Nouvelle-Écosse. Les $£ 50$ qu'il possédait lui permettraient d'être soigné à l'hôpital jusqu'au printemps, après quoi il faudrait subvenir à ses besoins. Les associés de la Caisse n'attireraient-ils pas ainsi sur eux les bénédictions du ciel en y pourvoyant ? ${ }^{33}$

$\mathrm{M}^{\mathrm{gr}}$ Plessis devint rapidement la plaque tournante de la Caisse, conséquence normale de la fonction de président que l'assemblée générale de 1801 lui attribua pour un temps illimité et qu'il conserva de fait durant tout son épiscopat jusqu'en 1825. Plusieurs prêtres dans le besoin s'adressaient directement à lui. Par ailleurs, il prenait lui-même l'initiative d'inviter des confrères à tirer profit de la Caisse. Il savait que ses administrateurs ne cherchaient pas à accumuler un gros capital, mais désiraient plutôt verser annuellement la majeure partie des cotisations reçues.

On note, par exemple, que $\mathbf{M}^{\mathrm{gr}}$ Plessis suggéra à GabrielAntoine Champion, missionnaire aux îles de la Madeleine, de se retirer à Halifax, ou -chez des confrères du Cap-Breton, ou encore à l'Hôpital général de Québec. "La Cảisse ecclésiastique vous fera une pension annuelle de $\$ 200.00$ sur laquelle vous pourrez compter et qui commencera à courir du moment que vous aurez cessé vos fonctions de missionnaire ». Champion se retira, de fait, chez François Le Jamtel, à Arichat; il y mourut l'année suivante. L'évêque invita le confrère accueillant à lui transmettre le compte des dépenses encourues par la maladie et la mort de ce prêtre missionnaire ${ }^{34}$.

L'évêque de Québec ne pouvait utiliser l'argent de la Caisse sans l'autorisation des procureurs élus par les membres. Par discrétion, il aurait parfois voulu cacher l'indigence d'un prêtre, même d'un missionnaire, aux yeux de ses confrères. Sous le couvert d'une allocation à une institution, par exemple à la mission de Carleton, il désirait aider Joseph-Marie Bellenger lui-même. Comme la demande de ce dernier lui était parvenue deux semaines trop tard, l'évêque envisagea d'utiliser dans ce but les arrérages des

33 AAQ, $C L$, V, p. 632, 2 octobre 1800.

34 AAQ, RL, VI, p. 46, 11 août 1807 ; IV, p. 257, 4 juin 1808. 
cotisations. Mais ce serait long, car il lui fallait tout de même consulter par écrit chacun des membres du Bureau, ce qui requérait au moins deux mois ${ }^{35}$.

On ne pouvait cependant pas assurer à un curé démissionnaire que la Caisse lui fournirait une pension suffisante pour continuer à tenir ménage ${ }^{36}$. Il paraissait donc inouï à $\mathbf{M}^{\text {gr }}$ Plessis qu'un membre de la Caisse eût marchandé d'avance ce qui lui serait versé en cas de maladie, de vieillesse ou d'infirmité. «On ne crie pas avant d'être battu ». La plus forte pension annuelle allouée à un prêtre jusqu'en 1817 s'éleva à $£ 70$. L'évêque s'opposait à ce qu'on résignât une cure où l'on faisait encore du bien, pour profiter d'une pension versée par la Caisse ${ }^{37}$. Le curé Joseph Verreau ne gagna pas moins son point, l'année suivante, en obtenant $£ 80$ de la Caisse ecclésiastique ; on était même prêt à augmenter ce montant s'il s'avérait vraiment insuffisant ${ }^{38}$.

La Caisse pourvut aussi aux besoins les plus pressants de Janvier Leclerc, curé au Cap-Santé. On lui nomma un successeur, lui versa $£ 50$ et l'invita à se retirer pendant un an chez son frère qui paraissait bien disposé à son égard ${ }^{39}$. Tout en étant assuré de l'aide de la Caisse pour défrayer les soins des religieuses et du médecin dans le cas de William Dullard, $\mathbf{M}^{\mathrm{gr}}$ Lartigue s'informa si la Caisse acquitterait aussi les frais funéraires; dans ce cas, le prêtre serait enterré de la même façon que ses confrères de Montréal ${ }^{40}$. Le président de la Société, $\mathbf{M}^{\mathrm{gr}}$ Plessis, acquiesça immédiatement à cette suggestion; il ne lui semblait pas raisonnable que cette Société abandonnât ses membres sur les bords de la fosse ${ }^{41}$.

\section{Aide de plus en plus diversifiée}

À mesure que les cotisations s'accrurent, les services rendus par la Caisse s'étendirent à d'autres champs d'action, en particulier du côté de l'éducation. Dès 1807 , un dénommé Boisseau remercia $\mathrm{M}^{\mathrm{gr}}$ Plessis du fait que la moitié des dépenses de son fils pour

35 Ibid., IX, p. 37, octobre 1816.

36 AAQ, CL, VI, p. 278, 6 octobre 1817, Panet à Plessis.

37 AAQ, $R L$, IX, p. 212, septembre 1817, à Joseph Verreau, curé à Saint-Roch des Aulnaies.

38 Ibid., p. 430, octobre 1818.

39 Ibid., p. 217, 23 septembre 1817, Plessis à Leclerc.

40 ACAM, $R L L$, II, p. 126,8 janvier 1823 , à Plessis.

41 ACAM, 295.101, 823-2, 16 janvier. 
son entretien au Collège de Nicolet serait défrayée par la Caisse ecclésiastique ${ }^{42}$. $\mathbf{M}^{\mathrm{gr}}$ Plessis engagea même le supérieur des sulpiciens et ses confrères à garder pour les étudiants du Collège de Montréal les montants d'argent dus à la Caisse ecclésiastique. Il pensait en effet que les procureurs de cette dernière "consentiraient peut-être difficilement à en faire l'application à des jeunes gens qui étudient à ce Collège ${ }^{43}$; il valait mieux que l'argent ne sortît pas du Collège.

Personne ne doutera que cette aide financière était d'autant plus motivée qu'elle s'adressait à des candidats éventuels au sacerdoce. Dans le cas des collégiens du Haut-Canada, l'intention était explicite. En effet, vers $1810, M^{\text {gr }}$ Plessis commença à faire payer par la Caisse les études de certains d'entre eux; auparavant, il prenait l'argent à même ses revenus personnels ${ }^{44}$. Les ecclésiastiques de cette province qui se préparaient au sacerdoce à Montréal, à Québec ou à Nicolet, profitaient déjà de bourses octroyées par la Caisse ${ }^{45}$. On incita cependant le vicaire général du Haut-Canada, Alexander Macdonell, à être membre de la Caisse ecclésiastique et à y payer ainsi sa contribution ${ }^{46}$. On donna dès lors, selon les années, $£ 32$ pour un séminariste écossais étudiant à Montréal ${ }^{47}$, $£ 4$ pour compléter la pension d'un autre au Séminaire de Québec 48 et $£ 40$ pour les étudiants écossais du Collège de Montréal ${ }^{49}$. Tous, collégiens et séminaristes, aspiraient à devenir prêtres pour le HautCanada.

Il est vrai que vers $1810-1815$ l'engouement pour les études classiques était fort grand. Les administrateurs de la Caisse SaintMichel y contribuèrent. L'école latine de Saint-Pierre, Rivière du Sud, reçut ainsi $£ 25^{50}$. Une autre école presbytérale, celle de Saint-François, toucha un montant similaire l'année suivante : « $£ 10$ pour le salaire de Binet, $£ 5$ pour aider les écoliers pauvres à se

\footnotetext{
42 AEP, Montmagny (St-Thomas), I, p. 83, 24 septembre 1807.

43 AAQ, RL, VII, p. 55, 23 octobre 1809, à Jean-Henry-Auguste Roux, supérieur des sulpiciens.

44 AAK (Archdiocesan Archives of Kingston), Bishop Alexander Macdonell's Correspondance, I, 4 mai 1810, Plessis à Macdonell.

45 Ibid., 25 septembre 1811, Extrait du Registre de la Société ecclésiastique de Saint-Michel.

46 Ibid., 4 mai 1810, Plessis à Macdonell.

47 Ibid., 25 septembre 1811.

48 Ibid., 26 septembre 1815.

49 AAQ, $R L$, VIII, p. 30, 4 février 1813, Plessis à Roux.

50 Ibid., VII, p. 21, 24 septembre 1809, Plessis à Michel Pâquet, curé.
} 
procurer le papier et les livres nécessaires pour l'étude du latin; $£ 5$ aux deux maîtres pour apporter quelques additions et améliorations à leur nourriture ». Les autres $£ 5$ étaient à la disposition du curé ${ }^{51}$. Une autre année, le Séminaire de Nicolet reçut $£ 100$ et l'école anglaise (probablement de Québec) $£ 5052$.

D'autres formes d'aide que celle de l'éducation avaient parfois leur place dans les sommes d'argent octroyées. On versa ainsi $£ 50$ à l'église du faubourg Saint-Roch ${ }^{53}$; on accepta de défrayer l'impression de certains livrets, comme La journée $d u$ chrétien en iroquois ${ }^{54}$ ou encore celle du Nouveau Testament en français ${ }^{55}$. Avant de partir pour l'Europe en 1819, $\mathrm{M}^{\mathrm{gr}}$ Plessis invita son coadjuteur à appuyer divers projets à la réunion suivante du Bureau : $£ 100$ pour la construction d'églises dans le HautCanada ; $£ 50$ pour le transport et le maintien de missionnaires en cette même province ; $£ 30$ pour le missionnaire de Percé; $£ 20$ pour la randonnée de Jean-Baptiste Kelly ou de Joseph-Edouard Morisset à Miramichi, le printemps suivant; $£ 100$ pour des séminaristes pauvres, ecclésiastiques ou écoliers, sans distinction de nationalité ; $£ 25$ ou $£ 50$ au Séminaire de Nicolet ("s'il reste de l'argent $)^{56}$. $\mathbf{M}^{\mathrm{gr}}$ Panet se demanda, une autre année, si la Caisse ecclésiastique ne pourrait pas aider $\mathbf{M}^{\mathrm{gr}}$ Provencher à défrayer son coûteux voyage de retour à la Rivière-Rouge ${ }^{57}$. Deux ans après avoir emprunté $£ 100$ à la Caisse (service qu'elle rendait aussi) pour la construction de l'église Saint-Jacques ${ }^{58}, \mathrm{M}^{\mathrm{gr}}$ Lartigue insista auprès de $\mathrm{M}^{\mathrm{gr}}$ Plessis pour qu'il amenât les autres membres du Bureau à les lui donner. " Je crois que votre influence ferait beaucoup pour cela et qu'il serait facile de faire entendre aux procureurs que mon œuvre est pour le moins aussi utile à la religion que plusieurs autres pour lesquelles ils ont libéralement

51 Ibid., p. 222, 17 nov. 1810, Plessis à Urbain Orfroy. Louis Binet avait été tonsuré le $1^{\text {er }}$ octobre 1809 , mais ne recevrait aucun ordre, ni mineur, ni majeur (AAQ, RI (Registres d'insinuations), G, 171r-v). Quand il s'engagea comme professeur à Saint-François, il pouvait encore porter la soutane.

52 AAQ, $C L$, VI, p. 209, 15 septembre 1815, Panet à Plessis.

53 AAQ, CL, VI, p. 209, 15 septembre 1815, Panet à Plessis.

54 AAQ, $R L$, VIII, p. 545, septembre 1816, à Joseph Marcoux, missionnaire à Saint-Régis.

55 Ibid., IX, p. 182, août 1817, à Roux.

56 AAQ, $C L$, VI, pp. 305-306, 1er juillet.

57 Ibid., p. 376, 10 avril 1822, à Plessis.

58 ASQ, Lettres $T$, p. 93, 6 octobre 1823, Ignace Bourget, secrétaire de Mgr Lartigue, à Narcisse-Charles Fortier, secrétaire de Mgr Plessis. 
donné 》59. La même année, l'auxiliaire de Montréal avait aussi demandé $£ 40$ pour le misssionnaire de la Petite-Nation ${ }^{60}$.

On constate que tout au long de son épiscopat $\mathrm{M}^{\mathrm{gr}}$ Plessis eut un mot très important à dire dans les allocations de la Caisse. Il prévenait souvent lui-même les destinataires de l'argent voté, surtout quand il s'agissait de subventions spéciales durant l'année. Il avertit ainsi Joseph Lefrançois, missionnaire à Paspébiac, que la Caisse avait de nouveau accordé un montant total $£ 30$ à lui et à son collègue de Percé, Charles Thomas Caron. Il lui suggéra de garder $£ 20$ pour lui-même et de transmettre les autres $£ 10$ à ce dernier ${ }^{61}$.

Sous $\mathrm{M}^{\mathrm{gr}}$ Panet, la Caisse semblait devoir continuer à rendre toutes sortes de services, atteignant ainsi l'ensemble des objectifs qu'avait initialement prévus $\mathbf{M}^{* r}$ Hubert. $\mathbf{M}^{\mathrm{rr}}$ Macdonell passa par $M^{\mathrm{gr}}$ Lartigue pour obtenir une subvention qui lui aurait permis de terminer la construction de trois petites églises à Cornwall, à Prescott et à Brockville ${ }^{62}$. Ce fut cependant peine perdue, car les fonds de la Caisse baissaient et un nouveau séminaire devait être bâti à Nicolet ${ }^{63}$. Il importait de soulager aussi le curé Antoine Girouard, fondateur du Collège de Saint-Hyacinthe. À cause des dettes ainsi contractées, $M^{\mathrm{gr}}$ Lartigue proposa à $\mathbf{M}^{\mathrm{gr}}$ Panet qu'on le relevât de sa créance de $£ 100$, due à un prêt déjà fait à la Caisse. "Personne ne mérite plus que lui de l'encouragement»64. Le Bureau y verrait la fois suivante ${ }^{65}$.

De cette façon, en 1827 , seulement deux prêtres avaient été aidés dans leurs besoins personnels. L'année suivante, la liste des subventions indique ceci : $£ 25$ à René Coyteux, prêtre infirme ; $£ 25$ au missionnaire de Drummondville ${ }^{66}$; £ 25 pour l'école catholique de Trois-Rivières; $£ 15$ pour l'école irlandaise des Ursulines à Québec; $£ 30$ pour le Collège de Saint-Hyacinthe ; $£ 57.6 .0$ pour le Séminaire de Nicolet ; $£ 10$ pour une école de Chambly ; $£ 100$ déjà prêtés, maintenant donnés au Collège SaintPierre de Chambly. Ce montant de $£ 287.6 .0$ équivalait presque

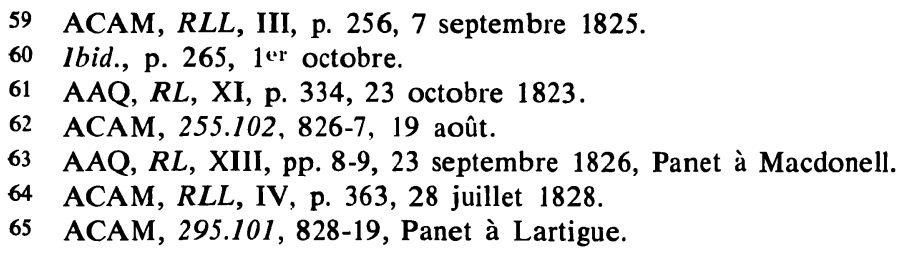


en totalité à la recette de l'année : $£ 291.14 .4$. Il restait tout de même un capital, puisqu'avant l'attribution des sommes mentionnées la Caisse possédait $£ 561.15 .11^{67}$.

$\mathrm{M}^{\mathrm{gr}}$ Lartigue se sentait ainsi à l'aise de suggérer aux administrateurs de la Caisse d'autres façons de soutenir des œuvres d'évangélisation. Il trouvait entre autres que les prêtres accepteraient plus facilement d'œuvrer dans une mission, si on les assurait d'un revenu suffisant. Les $£ 50$ allouées par le gouvernement et le peu que les Indiens donnaient n'offraient pas de garanties. Si la Caisse y ajoutait annuellement de $£ 30$ à $£ 40$, les missionnaires se sentiraient libérés de graves difficultés et opteraient plus aisément pour ce genre de travail apostolique ${ }^{68}$. Bien que ce projet ne se réalisât pas, $\mathrm{M}^{\mathrm{gr}}$ Lartigue en proposa un autre : subvenir aux besoins d'un prêtre, parlant anglais, qui parcourerait durant l'année longue les nombreux townships de son district; il s'y trouvait des milliers de catholiques sans secours spirituel et risquant d'apostasier ${ }^{69}$. L'évêque de Québec souligna que le missionnaire de Percé n'avait rien reçu cette année-là 70 ; l'année suivante on lui allouerait seulement $£ 15^{71}$, ce qui se poursuivrait comme tel d'ailleurs ${ }^{72}$.

Mais respectait-on vraiment le but premier de la Société ? Des prêtres ne se gênaient pas pour poser de temps à autre la question aux responsables de la Caisse. «Depuis grand nombre d'années, je cotise, paye et fournis à la Caissse ecclésiastique ; ... ne pourrait-elle pas m'aider en me donnant quelques subsides? L'institution de notre Caisse ecclésiastique est premièrement d'aider et de secourir nos membres ${ }^{73}$. On continuait, de fait, à privilégier l'objectif premier de la Caisse. Sans défrayer toutes les dépenses contractées à l'occasion d'une maladie (il ne s'agissait pas d'une assurance-maladie comme telle), on payait une pension à un prêtre

66 Jean Holmes avait reçu le même montant d'argent les deux années précédentes (AAQ, RL, XIII, p. 72, 1er décembre 1826, Plessis à J. Holmes, missionnaire à Drummondville) .

67 ACAM, 901.020, 828-2, 3 septembre, procès-verbal imprimé de la réunion annuelle, tenue à Yamachiche.

68 ACAM, RLL, III, 3 septembre 1825 , à Plessis.

69 Ibid., IV, p. 363, 28 juillet 1828, à Panet.

70 ACAM, 295.101, 838-19, Panet à Lartigue.

71 AAQ, $R L$, XIV, p. 120, 9 octobre 1829, Panet à John MacMahon.

72 Ibid., p. 486, 13 octobre 1831.

73 ACAM, 355.112, 828-3, 27 août, Laurent Aubry, curé au Sault-auRécollet, à Lartigue. 
qui délaissait l'exercice du ministère pour être soigné ${ }^{74}$.. On se fiait alors sur le rapport d'un médecin ou d'un autre prêtre ${ }^{75}$. On ne craignait pas de verser de l'argent à l'avance, même $£ 50$, pour l'année à venir. Si le pensionné se rétablissait plus vite que prévu et s'il reprenait un travail rémunéré, il lui fallait remettre le reste de la somme allouée ${ }^{76}$.

$M^{g r}$ Panet, bien que président de la Société, refusait d'outrepasser ses droits. Il l'a démontré dans le cas de Laurent Aubry. $\mathrm{M}^{\mathrm{gr}}$ Lartigue trouvait insuffisantes les $£ 60$ qui lui étaient allouées, bien qu'on eût entendu dire qu'il continuait à recevoir un tiers des dîmes de la cure à laquelle il avait résigné. L'auxiliaire de Montréal suggéra donc au président d'envoyer $£ 100$ à Aubry, quitte à ce qu'à sa réunion de l'année suivante le Bureau ratifiât cette décision de façon rétroactive ou même la modifiât. D'ailleurs, Joseph Verreau ne recevait-il pas $£ 80$ par année, tout en jouissant de son tiers sur Saint-Roch? De plus, Aubry avait servi le diocèse pendant quarante-sept ans et avait toujours payé fidèlement sa part à la Caisse ecclésiastique ${ }^{77}$. L'évêque de Québec se dit autorisé à avancer les deniers de la Caisse seulement dans le cas où l'un de ses membres devenait incapable d'exercer son ministère. Dans le cas d'Aubry, il fallait attendre la réunion suivante du Bureau pour qu'une augmentation fût allouée ${ }^{78}$. Ce fut différent quand démissionna le curé de Repentigny, Michel-Auguste Amyot, lui qui était membre de la Caisse depuis trente ans et longtemps procureur de cette dernière ${ }^{79} . \mathrm{M}^{\mathrm{gr}}$ Signay répondit à son collègue qu'il donnerait suite à une demande personnelle de la part de ce curé démissionnaire ${ }^{80}$. On peut noter que $\mathbf{M}^{\mathrm{gr}}$ Lartigue semblait porté à contrôler les versements de la Caisse pour ce qui concernait les prêtres de son district épiscopal, alors que $\mathbf{M}^{\mathrm{gr}}$ Panet et $\mathbf{M}^{\mathrm{gr}}$ Signay respectaient plus les administrateurs de la Caisse que ne l'avait fait leur prédécesseur $\mathbf{M}^{\mathrm{gr}}$ Plessis. $\mathrm{M}^{\mathrm{gr}}$ Lartigue avait appris à fonctionner avec ce dernier qui gérait la Caisse, semble-t-il, avec beaucoup d'autorité.

74 AAQ, $R L$, XIII, p. 442, 16 septembre 1828, Panet à Louis-Marie Lefebvre, curé à Saint-Laurent.

75 Ibid., XLV, p. 94, 9 septembre 1829, à Louis-Cuthbert RivardLoranger, curé à Saint-François d'Orléans.

76 ACAM, 295.101, 830-49, 10 novembre, Panet à Lartigue.

77 ACAM, RLL, VI, p. 127, 22 octobre 1831.

78 ACAM, 295.101, 831-57, 3 novembre, à Lartigue.

79 ACAM, RLL, VII, p. 12, 21 décembre 1832, à Panet.

80 ACAM, 295.101, 832-78, 26 décembre. 
Le nombre de prêtres pensionnés par la Caisse augmenta très rapidement au début de la décennie de 1830 . En 1834, on prévoyait ne plus pouvoir aider ni les missions, ni les dessertes, ni les maisons d'éducation, ni toute autre auvre de piété ${ }^{81} . M^{\mathrm{gr}}$ Lartigue trouvait fort normal que l'argent de la Caisse fût d'abord et avant tout destiné à assurer une subsistance aisée aux prêtres qui avaient usé leur vie en travaillant pour le diocèse. "La conduite contraire a déjà occasionné que plusieurs se sont retirés de cette association ${ }^{82}$. Il aurait aussi désiré que $\mathrm{M}^{\mathrm{gr}}$ Signay obtînt un indult de Rome, afin de pouvoir établir sur une cure de son choix une pension (un tiers des dîmes, par exemple) pour tout prêtre retiré, ayant travaillé au moins quinze ou vingt ans dans le diocèse. Il apparaissait absurde à $\mathbf{M}^{\mathbf{g r}}$ Lartigue que cette pension fût possible seulement à un curé qui avait œuvré les dix dernières années (ou plus) de son ministère au même endroit. Un curé opiniâtre et incompétent pouvait ainsi s'assurer une pension jusqu'à sa mort, tandis que le missionnaire ou le curé zélé qui avait rendu service à plusieurs endroits, sans avoir pu terminer une période de dix ans à sa dernière cure, ne profitait d'aucune pension de ce genre ${ }^{83}$. Malgré tout, les responsables de la Caisse allouèrent $£ 12.10$ à la mission de Matane, même cette année-là. Le vicaire de Rimouski y parcourait de trente à quarante lieues, au milieu de gens pauvres qui ne pouvaient rien donner à leur desservant. Outre ce montant d'argent, nécessaire pour circuler dans ces parages, le vicaire avait évidemment droit au lavage gratuit de son linge, à sa pension et à un salaire d'environ $£ 20$ à $£ 25$ par année, ce dont on avertit son curé ${ }^{84}$.

L'année suivante, $\mathrm{M}^{\mathrm{gr}}$ Lartigue se fit l'avocat des curés Louis Lamothe de Berthier et Laurent Aubry de Saint-Roch, tous deux en instance de résigner. Il lui semblait que les deux cures n'étaient pas assez riches pour les pourvoir d'un tiers de leurs revenus jusqu'à la mort de ces curés et qu'en conséquence la Caisse devrait assurer la subsistance de ces derniers. Gabriel-Léandre Arsenault envisageait lui aussi de demander une pension alimentaire à la Caisse, tout en travaillant un peu chez le curé Edouard-Joseph Crevier à SaintHyacinthe ${ }^{85}$. $\mathrm{M}^{\mathrm{gr}}$ Signay ne voyait pas pourquoi Louis Lamothe serait plus privé de son droit au tiers que tous les curés auxquels

81 Ibid., 834-28, 15 mai.

82 ACAM, $R L L$, VII, p. 473, 20 mai 1934, à Signay.

83 Ibid., pp. 171-172, 6 juillet 1833.

84 AAQ, RL, XVI, pp. 397-398, 9 décembre 1834, Signay à Thomas Féruce Destroismaisons.

85 ACAM, $R L L$, VII, pp. 766 et 773,15 et 25 août 1835 , à Signay. 
les évêques antérieurs avaient permis d'y prétendre ${ }^{86}$. Pourvoir les membres qui avaient besoin d'argent pour vivre continuait à prendre le dessus sur tout le reste ${ }^{87}$, ce qui s'accroîtrait, vu le nombre grandissant des prêtres démissionnaires ${ }^{88}$. La montée d'un jeune clergé fort nombreux permettait un renouvellement assuré du personnel.

\section{CONCLUSION}

Mais une nouvelle problématique surgit durant la décennie de 1830. Elle était déjà sous-jacente à plusieurs revendications de $\mathbf{M}^{\mathrm{gr}}$ Lartigue et de quelques prêtres de son district. À l'automne 1832, l'évêque auxiliaire se demanda comment on expliquait que seulement $£ 40$ avaient été attribuées à la région de Montréal, alors que $£ 201$ avaient été distribuées en tout. Comment comprendre que le Collège de Nicolet avait reçu $£ 56$ et que rien n'ait été octroyé à celui de Saint-Hyacinthe ${ }^{89}$ ? L'année suivante, $\mathrm{M}^{\mathrm{kr}}$ Lartigue eut droit à $£ 30$ pour l'ensemble des besoins pastoraux de son district, pendant que le reste du Bas-Canada en recevait $£ 120$. "Où est la juste proportion en faveur de mon district », s'écria-t-il, étant donné qu'il "fournit beaucoup plus à la Caisse que les trois vôtres ensemble et qu'il n'a pas les mêmes ressources ? \$ 90

Le partage semblait si disproportionné à chaque année que $\mathrm{M}^{\mathrm{rr} r}$ Lartigue pencha du côté d'un projet de constitution de Caisse ecclésiastique pour le district de Montréal. On y donna comme objectif unique «un secours suffisant dans le cas d'infirmité, de maladie ou de vieillesse ». On l'appela la Société ecclésiastique de Montréal, dédiée à saint Jean l'Évangéliste ${ }^{92}$, et elle perçut ses premières cotisations le $1^{\text {er }}$ août $1834{ }^{93}$.

86 ACAM, 295.101, 835-46, 31 août, à Lartigue.

87 Ibid., 835-48, 4 septembre, Signay à Lartigue.

88 AAQ, $R L$, XVII, pp. 459-460, 6 septembre 1836, Signay à CharlesVincent Fournier, curé à la Baie du Fèvre ; ASQ, Lettres $O$, p. 107, 10 août 1836, Jean-Denis Daulé, retiré à l'Ancienne Lorette, à Charles-François Baillargeon, curé de Québec et vice-trésorier de la Caisse ; ASQ, Lettres $S$, pp. 170 et 173,12 et 20 mai 1836, Signay et J.-D. Daulé à C.-F. Baillargeon.

89 ACAM, $R L L$, VI, pp. 474-475, 8 novembre, à Panet.

90 Ibid., VII, pp. 220-221, 9 septembre 1833, à Signay.

91 Ibid., p. 232, 14 septembre.

92 ACAM, 324.207, 834-1, texte des Règles de la Société ecclésiastique de Saint-Jean l'Évangéliste.

93 Ibid., 6 août. 
L'érection du diocèse de Montréal en 1836 promut l'importance de cette nouvelle Caisse ecclésiastique. La séparation d'avec celle de Québec ne s'opéra pas sans heurts, mais dès 1839-1840, elle s'avéra définitive.

Lucien LemieuX, prêtre L. Th., D. Hist. 